\title{
A Green Chemical Approach for the Synthesis of Gold Nanoparticles: Characterization and Mechanistic Aspect
}

\author{
Sujoy K. Das and Enrico Marsili* \\ School of Biotechnology, Dublin City University, \\ Collins Avenue, Dublin 9, Ireland
}

\begin{abstract}
This IRCSET-EMPOWER (Irish Research Council for Science, Engineering and Technology Postdoctoral Research Grant) project aims to improve current methodology for the synthesis of metal nanoparticles (NPs). The development of efficient methodology for metal nanomaterials synthesis is an economical and environmental challenge. While the current methods for NPs synthesis are often energy-intensive and involve toxic chemicals, NPs biosynthesis can be carried on at circumneutral $\mathrm{pH}$ and mild temperature, resulting in low cost and environmental impact. Nanomaterial biosynthesis has been already observed in magnetotactic bacteria, diatoms, and S-layer bacteria, however, controlled NPs biosynthesis is a relatively new area of research with considerable potential for development. A thorough understanding of the biochemical mechanism involved in NPs biosynthesis is needed, before biosynthetic methods can be economically competitive. The analysis and identification of active species in the nucleation and growth of metal NPs is a daunting task, due to the complexity of the microbial system. This project work focuses on the controlled biosynthesis of gold NPs by fungal microorganisms and aims to determine the biochemical mechanism involved in nucleation and growth of the particles.
\end{abstract}

Keywords Nanobiotechnology, Gold nanoparticles, Microbial synthesis, Living nanofactory, Green chemical approach.

${ }^{*}$ Corresponding author: Dr. Enrico Marsili,

Email: enrico.marsili@dcu.ie

Phone: 00353-1-7008515, Fax: 00353-1-7005412 


\section{Introduction}

The synthesis of metal nanoparticles (NPs) is a growing area of research in materials science because they exhibit unique properties (Gratzel 2001; Xia et al. 2003), different from those of bulk metals due to their unique size and shape dependent characteristics. Because of stability, oxidation resistance, and biocompatibility, gold NPs find wide applications in electronics and photonics, catalysis, information storage, chemical sensing and imaging, drug delivery and biological labeling (Elghanian et al. 1997; Cao et al. 2002). For each application, NPs of different size and shape are needed (Jin et al. 2001; Sun and Xia 2002), thereby synthetic protocols for the production of size and shape controlled monodisperse NPs are required. Since Faraday's pioneering work in 1857 on the synthesis of colloidal gold by reducing $\mathrm{NaAuCl}_{4}$ with a solution of phosphorus in carbon disulphide (Fig. 1) (The Royal Institution of Great Britain 2008; Thompson 2007), several physical and chemical methods have been developed to produce gold NPs. Synthetic techniques based on the reduction of metal ions with sodium citrate or sodium borohydride, followed by surface modification of the produced particles with suitable capping ligands and organic solvents (Niemeyer et al. 1998; Lévy et al. 2004), raised environmental concerns, because of the toxic compounds used in the process. Also, it is difficult to obtain NPs of defined size and shapes (e.g. spheres, rod, cubes, hexagons, etc.) in high yield. Current synthetic methods result in mixed-shape NPs that require expensive and low-yield purification procedures, such as differential centrifugation (Murphy 2002). These limitations invite new eco-friendly ("green chemistry”) methodology for production of nanocrystals with desired shape.

Natural processes such as biomineralization may be mimicked to design efficient NP synthesis techniques. Biomineralization processes exploit biomolecular templates that interact with the inorganic material throughout its formation resulting in the synthesis of particles with defined shape and size (Mann 1993). Bones, teeth and shells are typical examples of structural 
materials produced by natural biomineralization processes. A general scheme for NP synthesis in microorganisms is illustrated in Figure 2.

The adoption of biomineralization methods in the synthesis of nanostructured materials is expected to yield novel and more complex structural entities compared to those obtained with conventional methods (Brown et al. 2000; Klaus et al. 1999; Mukherjee et al. 2001; Xie et al. 2007a,b). Both uni and multicellular microorganisms are reported to produce inorganic nanomaterials either intra- or extracellularly. Some well-known examples (Mann et al. 1990; Oliver et al. 1995; Sleytr et al. 1999) of microbial mediated synthesis of inorganic materials include magnetotactic bacteria Magnetospirillum magnetotacticum (which synthesize magnetite NPs), S-layer bacteria Synechococcus sp., Bacillus stearothermophilus (which produce gypsum and calcium carbonate layers), and diatoms Coscinodiscus sp., Cylindrotheca fusiformis (which synthesize siliceous materials). However, the use of microorganisms in the deliberate and controlled NP synthesis is a new area of research.

\subsection{Bacteria in nanoparticle synthesis}

Among the microorganisms, prokaryotic bacteria have received the most attention in the area of NP biosynthesis. Beveridge and Murray (1980) have demonstrated that gold NPs readily precipitate in bacterial cells following incubation of the cells with $\mathrm{Au}^{+3}$ ions under ambient temperature and pressure. Organic phosphate compounds play a role in the in vitro development of octahedral $\mathrm{Au}$, possibly as bacteria-Au complexing agents. $\mathrm{Fe}^{+3}$ reducing bacteria Shewanella algae can reduce $\mathrm{Au}^{+3}$ ions in anaerobic environments. In the presence of S. algae and hydrogen gas, the Au ions are completely reduced and 10-20 nm gold NPs are formed (Konishi et al. 2004). Klaus-Joerger and co-workers (Klaus-Jorger et al. 1999) have demonstrated that Pseudomonas stutzeri AG259 isolated from a silver mine reduces $\mathrm{Ag}^{+}$ions and forms silver NPs of well-defined size and morphology, ranging from few a nm to $200 \mathrm{~nm}$ or more, within the periplasmic space. 


\subsection{Fungi in nanoparticle synthesis}

The use of fungi in the synthesis of NPs is a relatively recent addition and hold promises for large scale NP production. In fact, fungi secrete large amounts of the enzymes involved in NP synthesis and are simpler to grow both in the laboratory and at industrial scale. Different fungal and actinomycete species, i.e. Fusarium oxysporum, Verticillium sp., Thermomonospora sp., Rhodococcus sp. have been reported (Ahmad et al. 2003; Mandal et al. 2006) to synthesize NPs intra- or extracellularly. Shankar et al. (2004) synthesized gold nanoplates by fungal extracts. A brief over view on microbial synthesis of metal NPs is given in Table 1.

\subsection{Mechanism of nanoparticle synthesis}

While a large number of microbial species are capable of producing metal NPs, the mechanism of nanoparticle biosynthesis has not been established. The metabolic complexity of viable microorganisms complicates the analysis and identification of active species in the nucleation and growth of metal NPs. Recent works by Xie et al. (2007a) demonstrated that proteins are the principal biomolecules involved in the algal synthesis of gold NPs. Other researchers (Ahmad et al. 2003; He et al. 2007) have postulated that microorganisms secrete enzymes which may be responsible for the reduction of metal ions which result in the NPs nucleation and growth. Ahmad et al. (2003) postulated that a NADH-dependent reductase is involved in Ag NPs synthesis by Fusarium oxysporum. However, the biochemical mechanism of metal ion reduction and subsequent NP formation remains unexplored.

The elucidation of the biochemical pathways leading to gold biomineralization is necessary to develop a rational approach to NP biosynthesis. A number of issues need to be addressed from the nanotechnology and microbiological points of view before such biosynthetic procedures can compete with the conventional protocols. 
Preliminary experiments (Fig. 3) carried out in our laboratory demonstrated the synthesis of single crystal gold NPs from $\mathrm{HAuCl}_{4}$ when incubated with mycelia or cell-free extract of Rhizopus oryzae (Das et al. 2008; Das et al. 2009).

'Green chemistry' aims to employ environmentally benign solvents and nontoxic chemicals in synthetic methods, thereby reducing their environmental impact (Anastas and Warner 1998). This IRCSET-EMPOWER project will adopt such 'green chemistry' approach to synthesize gold NPs using microorganisms as a ‘living nano-factory’ by avoiding any chemical agents, to determine the biochemical mechanisms involved in the biomineralization process, and to isolate and purify the enzymes involved in the NP formation.

\section{Methodological approaches}

The present project work aims to i) synthesize gold NPs through room temperature reduction of gold ions by $R$. oryzae; and ii) determination of the biochemical mechanism involved in the nucleation and growth of gold NPs. $R$. oryzae will be grown in the laboratory as previously described (Das et al. 2009) and the cell-free extract will be prepared from $R$. oryzae mycelia following harvesting from the growth medium. The cell-free extract will then be used for the synthesis of gold NPs.

The NP will be characterized through Transmission Electron Microscopy (TEM), Atomic Force Microscopy (AFM), X-ray photoelectron spectroscopy (XPS), UV-visible and Fourier Transform Infrared spectroscopy (FTIR). The crystal structure of the NPs will be determined with the selected area electron diffraction (SAED) pattern obtained from TEM image.

The biochemical pathway involved in the NP biosynthesis will be assessed using heat inactivated or metabolically inhibited microorganisms (e.g., through sodium cyanide, formaldehyde, and 2,4-dinitrophenol) as control experiment. The enzymes responsible for reduction of $\mathrm{Au}^{+3}$ to $\mathrm{Au}^{0}$ will be obtained from the cell-free extract through several purification steps: ammonium sulphate fractionation, anion-exchange chromatography, chromatofocusing, 
and gel filtration. The purified enzyme(s) will then be used for the synthesis of gold NPs as described above. Kinetic measurements of enzyme activity for gold NP synthesis will then be undertaken at the optimal $\mathrm{pH}$ and temperature, as determine in separate experiments. The shape controlled synthesis of gold NPs by purified enzyme(s) will be also performed using varying reaction conditions, such as the concentration of gold ions, the $\mathrm{pH}$ of the solution, and the incubation period. All these conditions control the crystal growth kinetics and final NP morphology. Since the enzyme(s) secreted by $R$. oryzae act both as reducing and capping agent, the adsorption of such enzyme(s) on the growing crystals and their reducing activity changes with the above mentioned conditions, thereby resulting in different crystal shapes.

\section{Impact of the proposed research}

Metal NPs are relevant to numerous emerging technologies. The development of high yield and low cost methods for NP production is therefore an important challenge. Current methods for metal NP production require harsh chemicals and energy-intensive processes. It is consequently important to develop an eco-friendly sustainable ("green chemistry") alternative to the existing chemical methods. The microbial-mediated synthesis of metal NPs may replace some of the current physical and chemical methods in use for NP production. However, several issues need to be addressed before such biosynthetic procedures can compete with established protocols. Comprehension of biochemical mechanism involved in gold nanoparticle formation is crucial to the development of innovative and low-energy NPs production processes. This IRCSET-EMPOWER project deals with pioneer research on the synthesis of gold nanostructures employing microorganism simultaneously as the reducing and capping agent. This environmental-friendly methodology may be applied in various pharmaceutical and biomedical formulations, as well as in cellular imaging, biosensing, and drug delivery.

\section{Acknowledgement}

Funding source: IRCSET-EMPOWER 2009 Postdoctoral Research Grant. 


\section{References}

Ahmad A, Mukherjee P, Senapati S, Mandal D, Khan MI, Kumar R, Sastry M (2003) Extracellular biosynthesis of silver nanoparticles using the fungus Fusarium oxysporum. Colloids Surf B: Biointerfaces 28:313-318

Anastas PT, Warner JC (1998) Green Chemistry: Theory and Practice, Oxford University Press: New York, pp 30

Beveridge TJ, Murray RGE (1980) Site of metal deposition in the cell wall of Bacillus subtilis. J Bacteriol 141:876-887

Brown S, Sarikaya M, Johnson EA (2000) Genetic Analysis of Crystal Growth. J Mol Biol 299:725-735

Cao YC, Jin R, Mirkin CA (2002) Nanoparticles with Raman Spectroscopic Fingerprints for DNA and RNA Detection. Science 297:1536-1540

Das SK, Das AR, Guha AK (2009) Gold Nanoparticles: Microbial Synthesis and Application in Water Hygiene Management. Langmuir 25:8192-8199

Das SK, Das AR, Guha AK (2008) Synthesis of Gold Nanoparticles: A Green Chemical Approach. International Conference on Soft System ICSS-2008, Kolkata, India, February 3-15

Elghanian R, Storhoff JJ, Mucic RC, Letsinger RL, Mirkin CA (1997) Selective Colorimetric Detection of Polynucleotides Based on the Distance-Dependent Optical Properties of Gold Nanoparticles. Science 277:1078-1081

Gratzel M (2001) Photoelectrochemical cells. Nature 414:338-344

He S, Guo Z, Zhang Y, Zhang S, Wang J, Gu N (2007) Biosynthesis of gold nanoparticles using the bacteria Rhodopseudomonas capsulate. Mater Lett 61:3984-3987

Jin R, Cao Y, Mirkin CA, Kelly KL, Schatz GC, Zheng JG (2001) Photoinduced Conversion of Silver Nanospheres to Nanoprisms. Science 294:1901-1903

Klaus T, Joerger R, Olsson E, Granqvist C-G (1999) Silver-based crystalline nanoparticles, microbially fabricated. Proc Natl Acad Sci USA 96:13611-13614

Konishi Y, Nomura T, Tsukiyama T, Saitoh N (2004) Microbial preparation of gold nanoparticles by anaerobic bacterium. Trans Mater Res Soc Jpn 29:2341-2343

Lévy R, Thanh NTK, Doty RC, Hussain I, Nichols RJ, Schiffrin DJ, Brust M, Ferning DG (2004) Rational and combinatorial design of peptide capping ligands for gold nanoparticles. J Am Chem Soc 126:10076-10084

Mandal D, Bolander ME, Mukhopadhyay D, Sarkar G, Mukherjee P (2006) The use of microorganisms for the formation of metal nanoparticles and their application Appl Microbiol Biotechnol 69:485-492 
Mann, S (1993) Molecular tectonics in biomineralization and biomimetic materials chemistry. Nature 365:499-505

Mann S, Sparks NHC, Frankel RB, Bazylinski DA, Jannasch HW (1990) Biomineralization of ferrimagnetic greigite $\left(\mathrm{Fe}_{3} \mathrm{O}_{4}\right)$ and iron pyrite $\left(\mathrm{FeS}_{2}\right)$ in a magnetotactic bacterium. Nature 343:258-260

Mukherjee P, Ahmad A, Mandal D, Senapati S, Sainkar SR, Khan MI, Parischa R, Ajayakumar PV, Alam M, Kumar R, Sastry M (2001) Fungus mediated synthesis of silver nanoparticles and their immobilization in the mycelial matrix: a novel biological approach to nanoparticle synthesis. Nano Lett 1:515-519

Murphy CJ (2002) Nanocubes and Nanoboxes. Science 298:2139-2141

Nair B, Pradeep T (2002) Coalescence of nanoclusters and formation of submicron crystallites assisted by Lactobacillus strains. Cryst Growth Des 2:293-298

Niemeyer CM, Burger W, Peplies I (1998) Covalent DNA-Streptavidin Conjugates as Building Blocks for Novel Biometallic Nanostructures. Angew Chem Int Ed 37:2265-2268

Oliver S, Kupermann A, Coombs N, Lough A, Ozin GA (1995) Lamellar aluminophosphates with surface patterns that mimic diatom and radiolarian microskeletons Nature 378:47-50

Shankar SS, Rai A, Ankamwar B, Singh A, Ahmad A, Sastry M (2004) Biological synthesis of triangular gold nanoprisms. Nature Mat 3:482-488

Shankar SS, Ahmad A, Parischa R, Sastry M (2003) Bioreduction of chloroaurate ions by geranium leaves and its endophytic fungus yields gold nanoparticles of different shapes. $\mathrm{J}$ Mater Chem 13:1822-1826

Sleytr UB, Messner P, Pum D, Sara M (1999) Crystalline bacterial cell surface layers (S layers): from supramolecular cell structure to biomimetics and nanotechnology. Angew Chem Int Ed 38:1035-1054

Sun Y, Xia Y (2002) Shape-Controlled Synthesis of Gold and Silver Nanoparticles Science 298:2176-2179

The Royal Institution of Great Britain, cited 18 Dec. 2008; available from: http://www.rigb.org/rimain/heritage/faradaypage.jsp

Thomson, D (2007) Michael Faraday's Recognition of Ruby Gold: the Birth of Modern Nanotechnology. Gold Bulletin 40: 267-269

Xia, Y. et al. (2003) One-Dimensional Nanostructures: Synthesis, Characterization, and Applications. Adv Mater 15:353-389

Xie J, Lee JY, Wang DIC, Ting YP (2007a) Silver Nanoplates: From Biological to Biomimetic Synthesis. ACS Nano 1:429-439 
Xie J, Lee JY, Wang DIC, Ting YP (2007b) High-Yield Synthesis of Complex Gold Nanostructures in a Fungal System. J Phys Chem C 111:16858-16865 


\section{Legend to Figures}

Figure 1: Faraday's colloidal suspension of gold (A) [The Royal Institution of Great Britain, 2008]; High resolution transmission electron microscopic image (B) of individual colloidal gold particles (at a magnification of $10^{7} \times$ ), prepared according to Faraday's recipe [Edwards, Thomas 2007].

Figure 2: Schematic of biomineralization process for nanoparticle synthesis.

Figure 3: Atomic force microscopic images (A-B) of gold nanoparticles synthesized on the surface of the fungal mycelia (A, low resolution; B, high resolution); High resolution transmission electron microscopic image (C) shows that the average particles size is $20 \mathrm{~nm}$; Selected area electron diffraction (SAED) pattern (D) indicates that the synthesized gold nanoparticles are single crystal. 
Table 1: Synthesis of nanoparticles by different microorganisms

\begin{tabular}{lll}
\hline Microorganisms & Metal nanoparticle & References \\
\hline $\begin{array}{l}\text { Bacteria } \\
\text { Bacillus subtilis }\end{array}$ & Gold & Beveridge and Murray 1980 \\
$\begin{array}{l}\text { Shewanella algae } \\
\text { Pseudomonas stutzeri }\end{array}$ & Gold & Konishi et al. 2004 \\
Lactobacillus & Gold, silver, Au-Ag alloy & Klaus et al. 1999 \\
$\begin{array}{l}\text { Escherichia coli } \\
\text { Rhodococcus }\end{array}$ & Gold & Brown et al. 2002 \\
Fungi & Gold & He et al. 2007 \\
Verticillium & & \\
Fusarium oxysporum & Gold, silver & Mukherjee et al. 2001 \\
Colletotrichum sp. & Gold, silver, Au-Ag alloy & $\begin{array}{l}\text { Ahmad et al. 2003; Mandal et } \\
\text { al. 2006; and there in }\end{array}$ \\
\hline
\end{tabular}




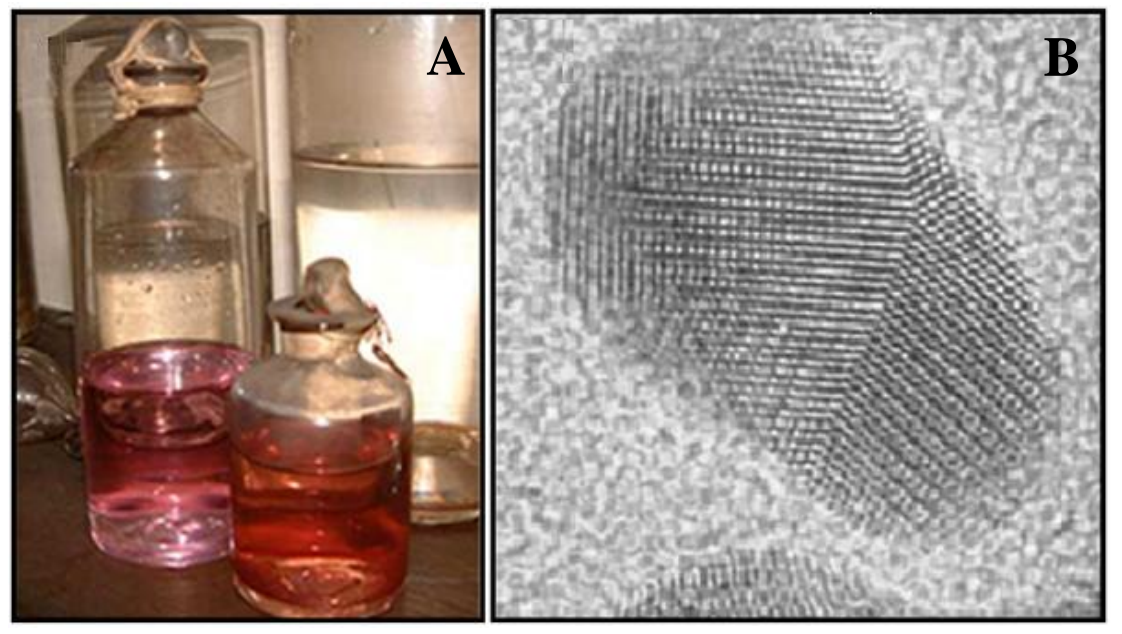

Figure 1 


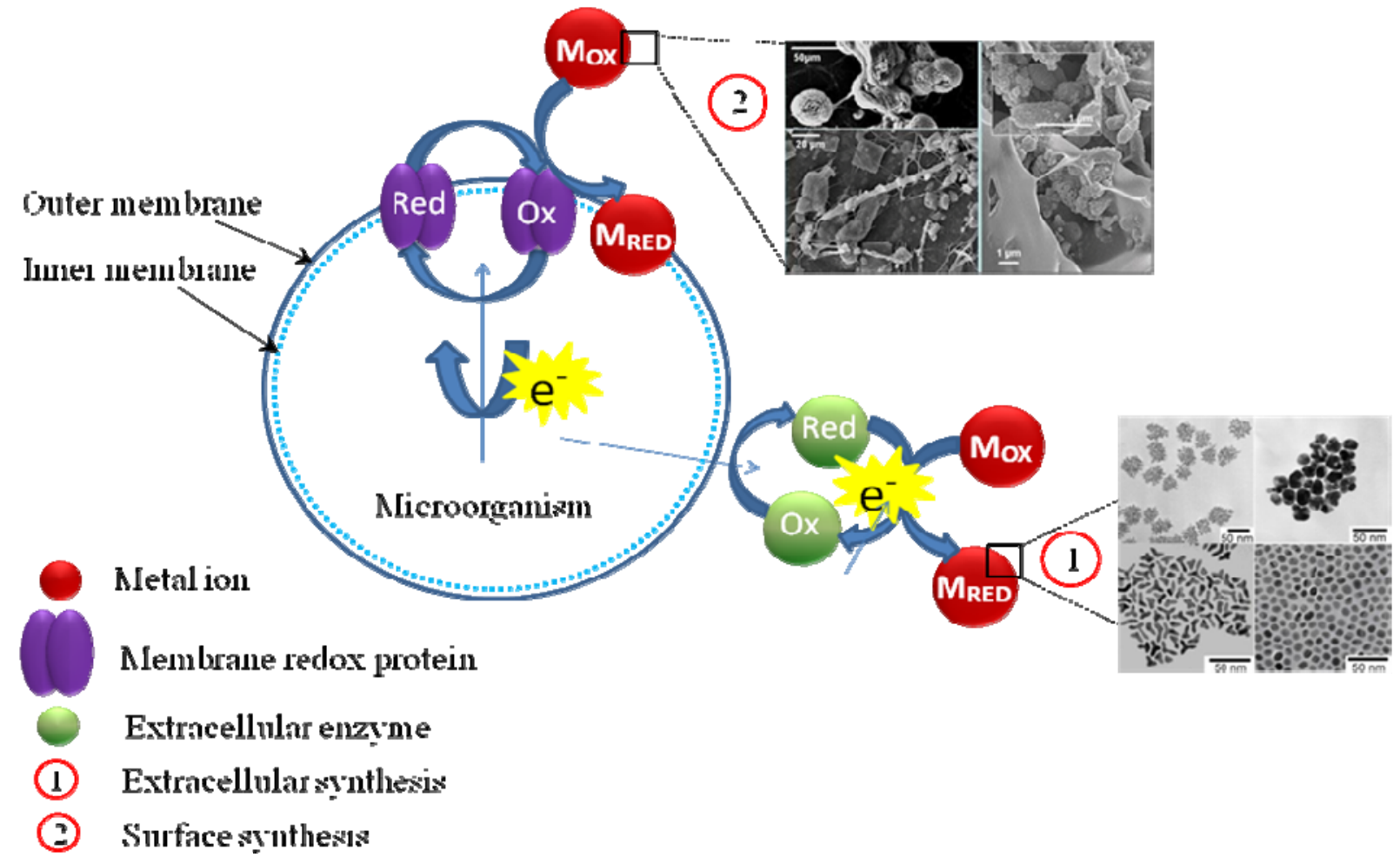

Figure 2 

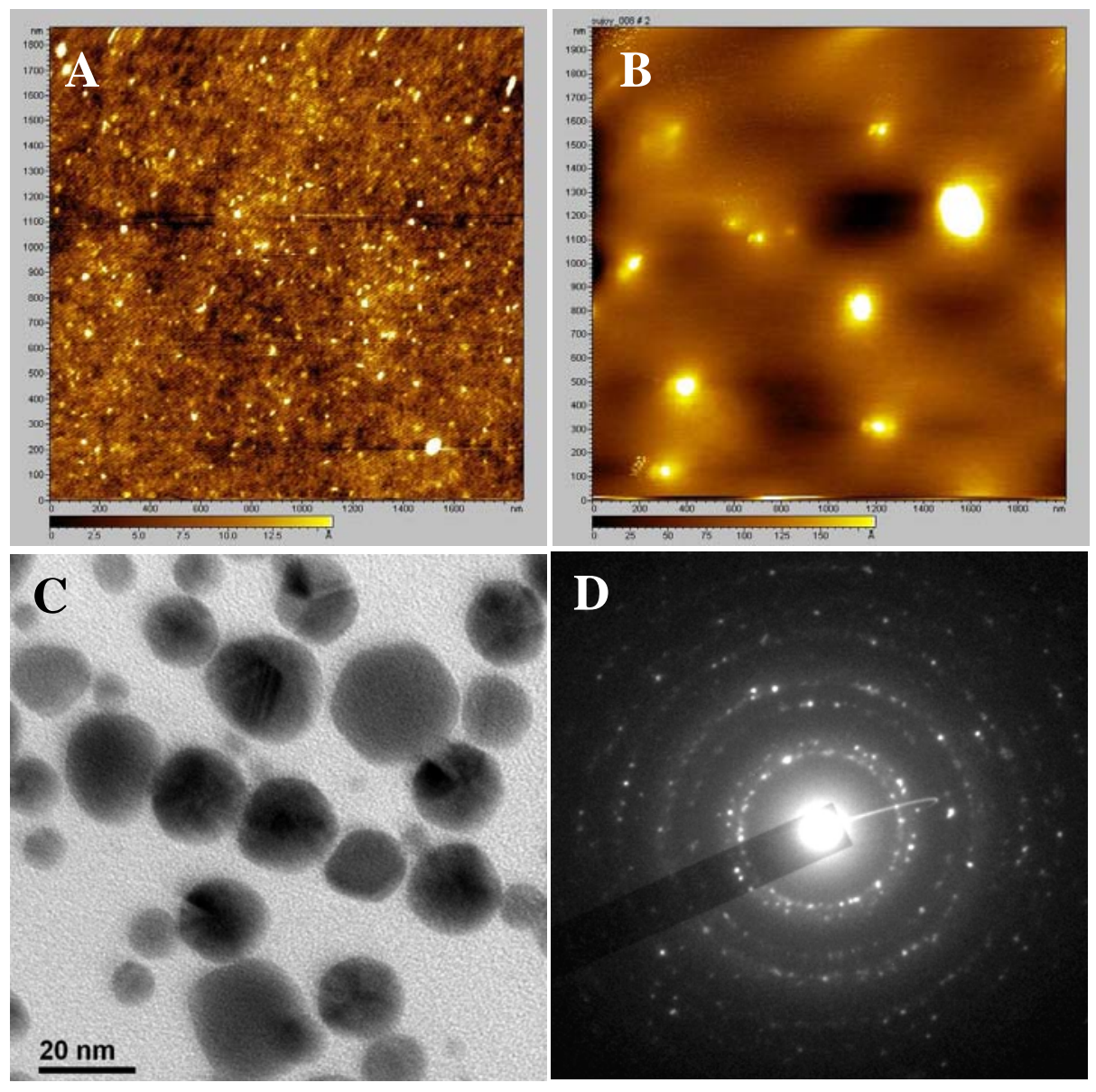

Figure 3 